\section{Buchrezension zu: Basiswissen Pharmakologie}

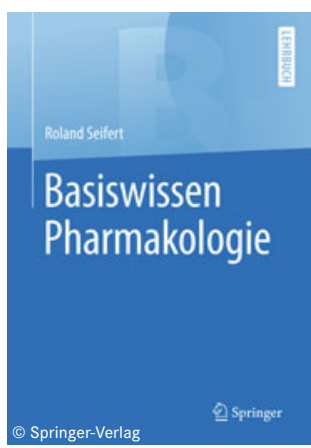

Basiswissen Pharmakologie Roland Seifert

584 S., Springer-Verlag, 2. Aufl., 2021. SC., 39,99€.

ISBN: 9783662605035

Auch als E-Book erhältlich

DOI: 10.1007/s12268-021-1655-8 (C) Der Autor 2021

Das Fach Pharmakologie und Toxikologie ist im Studium der Humanmedizin von zentraler Bedeutung, da es für alle klinischen Fächer von Relevanz ist. Bei den mehr als 10.000 Arzneistoffen, die sich auf dem deutschen Markt befinden, ist eine solide pharmakologische Grundlage notwendig, um den Überblick zu behalten und um die wissenschaftliche und fachliche Kompetenz für die Beurteilung und die Verordnung von Arzneimitteln bei der zukünftigen Berufsausübung zu haben. Studierende der Medizin stehen angesichts der Menge an Arzneistoffen aber gerade zu Beginn ihrer klinischen Ausbildung oftmals vor einer großen Hürde, die es zu überwinden gilt. Diese Hürde kann leichter genommen werden, wenn sich auf Basis von physiologischen und pathophysiologischen Mechanismen die Wirkprinzipien von Arzneistoffgruppen ableiten oder erklären lassen.

Vor diesem Hintergrund hat der Autor das Lehrbuch „Basiswissen Pharmakologie“ geschrieben, welches nun in der 2. Auflage erschienen und um 90 Seiten dicker geworden ist. Das didaktische Grundprinzip ist gleichgeblieben: Geschickt baut der Autor auf das vorhandene (patho-)physiologische Vorwissen der Studierenden auf und versucht damit konsequent und verständlich Wirkmechanismen von Arzneistoffen abzuleiten und zu erklären. Auch der Aufbau der einzelnen Kapitel hat sich nicht geändert: Nach einer Kurzeinleitung folgen die wichtigsten Merksätze und der eigentliche Fließtext, in den Tabellen, Abbildungen, Merksätze, Fallbeispiele und Übungsfragen eingebettet sind. Der Verzicht auf den bläulichen Hintergrund und die Verwendung einer etwas größeren Schrift in den Abbildungen haben die Lesbarkeit deutlich verbessert.

Dieses Lehrbuch ist in $37 \mathrm{Kapi}-$ tel untergliedert, welche vier übergeordneten Themenbereichen (Allgemeine Prinzipien, Integrative Systeme, Pharmakotherapie und Prüfungsteil) zugeordnet sind. In der nun vorliegenden 2. Auflage wurden alle Kapite nicht nur aktualisiert, sondern zum Teil auch deutlich erweitert, wie z. B. das Kapitel Pharmakodynamik, in welches nun unter anderem ein Abschnitt über typische Erkennungssilben von Arzneistoffen integriert wurde. Dies erleichtert es dem Studierenden, einzelne Arzneistoffe anhand des Namens den entsprechenden Arzneistoffgruppen zuzuordnen.

Aus Platzgründen wurde das Kapitel Antidementiva gestrichen. Begrenzungen und Vereinfachungen können aber auch nachteilig sein. So ist z. B. die Verwendung der zahlreichen Abkürzungen beim konzentrierten Lesen manchmal hinderlich, da diese Abkürzungen im Abkürzungsverzeichnis nachgeschlagen werden müssen. Die vielen einfachen $A b-$ bildungen hingegen erleichtern es, dass Wirkmechanismen gut verstanden und einfach gelernt werden können.

Dieses kompakte Lehrbuch ist weder ein knappes stichpunktartiges Nachschlagewerk bzw. eine Kurzübersicht über das Wissensgebiet noch ein vollumfassendes
Lehrbuch. Es bietet aber die Möglichkeit eines leichten, dennoch ausführlich genug gehaltenen Einstiegs in das Gebiet der Pharmakologie und Toxikologie. Für Studierende der Medizin ist es in bester Weise geeignet, um sich beim Lernen auf die wesentlichen pharmakologischen Schwerpunkte zu fokussieren.

Wie schon in der Erstausgabe geschehen, erfolgte auch bei dieser Auflage eine Anlehnung an die aktuelle Version des nationalen Lernzielkatalogs Medizin (NKLM 2.0), was für die Studierenden der Medizin von Vorteil ist. Leider wurden bei den Multiple-Choiceund Fallfragen viele Fragen aus der Erstauflage verwendet. Hier würde man sich auch eine Aktualisierung in Form neuer Fragen wünschen. Zudem eignet es sich als Lehrbuch für andere Studiengänge, wie z. B. der Pharmazie und der Zahnmedizin, in denen das Fach Pharmakologie und Toxikologie von Bedeutung ist.

Zusammenfassend ist es dem Autor gelungen, seine sehr interessante Erstauflage nochmals deutlich zu verbessern. Es ist ein anspruchsvolles Lehrbuch entstanden, das seinem Anspruch einer gut zugänglichen und daher leicht verständlichen Vermittlung pharmakologischen Basiswissens mehr als gerecht wird.

Klaus Höcherl,

Institut für medizinische und pharmazeutische Prüfungsfragen Mainz, khoecherl@impp.de

Diese Rezension erscheint Open Access.
* Funding note: Open Access funding enabled and organized by Projekt DEAL. Open Access: Dieser Artikel wird unte der Creative Commons Namensnennung 4.0 International Lizenz veröffentlicht, welche die Nutzung, Vervielfältigung, Bearbeitung, Verbreitung und Wiedergabe in jeglichem Medium und Format erlaubt, sofern Sie den/die ursprünglichen Autor(en) und die Quelle ordnungsgemäß nennen, einen Link zur Creative Commons Lizenz beifügen und angeben, ob Änderungen vorgenommen wurden. Die in diesem Artikel enthaltenen Bilder und sonstiges Drittmaterial unterliegen ebenfalls der genannten Creative Commons Lizenz, sofern sich aus der Abbildungslegende nichts anderes ergibt. Sofern das betreffende Material nicht unter der genannten Creative Commons Lizenz steht und die betreffende Handlung nicht nach gesetzlichen Vorschriften erlaubt ist, ist für die oben aufgeführten Weiterverwendungen des Materials die Einwilligung des jeweiligen Rechteinhabers einzuholen. Weitere Details zur Lizenz entnehmen Sie bitte der Lizenzinformation auf

http://creativecommons.org/licenses/ by $/ 4.0 /$ deed.de. 